\title{
On the Heaviest Increasing or Decreasing Subsequence of a Permutation, and Paths and Matchings on Weighted Point Sets
}

\author{
Toshinori Sakai ${ }^{1}$ and Jorge Urrutia ${ }^{2}$ \\ 1 Tokai University, Shibuya-ku, Tokyo, Japan, \\ sakai@tokai-u.jp \\ 2 Universidad Nacional Autónoma de México, México D.F., México, \\ urrutia@matem.unam.mx
}

\begin{abstract}
Let $S=\{s(1), \ldots, s(n)\}$ be a permutation of the integers $\{1, \ldots, n\}$. A subsequence of $S$ with elements $\left\{s\left(i_{1}\right), \ldots, s\left(i_{k}\right)\right\}$ is called an increasing subsequence if $s\left(i_{1}\right)<\cdots<s\left(i_{k}\right)$; It is called a decreasing subsequence if $s\left(i_{1}\right)>\cdots>s\left(i_{k}\right)$. The weight of a subsequence of $S$, is the sum of its elements. In this paper, we prove that any permutation of $\{1, \ldots, n\}$ contains an increasing or a decreasing subsequence of weight greater than $n \sqrt{2 n / 3}$.

Our motivation to study the previous problem arises from the following problem: Let $P$ be a set of $n$ points on the plane in general position, labeled with the integers $\{1, \ldots, n\}$ in such a way that the labels of different points are different. A non-crossing path $\Pi$ with vertices in $P$ is an increasing path if when we travel along it, starting at one of its end-points, the labels of its vertices always increase. The weight of an increasing path, is the sum of the labels of its vertices. Determining lower bounds on the weight of the heaviest increasing path a point set always has.

We also study the problem of finding a non-crossing matching of the elements of $P$ of maximum weight, where the weight of an edge with endpoints $i, j \in P$ is $\min \{i, j\}$.
\end{abstract}

\section{Introduction}

Let $n$ be a positive integer. Consider any permutation $S$ of the integers $\{1, \ldots, n\}$. A well known result of Erdös and Szekeres [4], asserts that $S$ always contains an increasing or a decreasing subsequence with at least $\lceil\sqrt{n}\rceil$ elements. The weight of a subsequence of a permutation $S$ is the sum of its elements. For example if we consider the permutation:

$$
5,2,8,1,7,4,3,6,
$$

the weight of the increasing subsequence $2,4,6$ is equal to 12 . In this paper we study the problem of finding the heaviest increasing, or decreasing subsequence of a permutation. For the permutation considered before, the heaviest increasing, or decreasing subsequence, consists of $8,7,4,3$ and has weight 22 . 
We prove that any permutation of $\{1, \ldots, n\}$ always has an increasing or a decreasing subsequence with weight greater than $n \sqrt{n / 3}$, our bound is asymptotically tight. Our solution is somehow related to a well known problem of Tutte [8] involving the efficient packing of squares of different sizes into a rectangle, or a square of small area. The permutations giving the tight bound for our problem, produce efficient packings of squares with areas $1^{2}, 2^{2}, \ldots, n^{2}$ under some conditions.

A finite sequence $a_{1}, a_{2}, \ldots, a_{k}$ is said to be unimodal (resp. anti-unimodal) if there is an $m, 1 \leq m \leq k$, such that $a_{1}<a_{2}<\cdots<a_{m}$ and $a_{m}>a_{m+1}>$ $\cdots>a_{k}$ (resp. $a_{1}>a_{2}>\cdots>a_{m}$ and $a_{m}<a_{m+1}<\cdots<a_{k}$ ). We also study the problem of finding a heavy unimodal or anti-unimodal subsequence of a permutation of $\{1, \ldots, n\}$. We show that any permutation of $\{1, \ldots, n\}$, always has a unimodal or anti-unimodal subsequence of weight greater than $n \sqrt{2 n / 3}$.

Let $P$ be a set of $n$ points on the plane in general position which are labeled with $\{1, \ldots, n\}$. Our results are motivated by the study of longest or heaviest non-crossing increasing paths connecting elements of $P$. In particular, as we will see in Section 2, for labeled point sets in convex position, finding a noncrossing increasing path with maximum weight, is related to that of finding a heaviest unimodal or anti-unimodal subsequence in some permutation. The problem of finding non-crossing paths, cycles and trees with vertices on point sets on the plane optimizing some given functions, has been of interest to many computational geometers for some time now. Alon, Rajagopalan, and Suri [1], and Dumitrescu and Tóth [3] studied the problem of finding non-crossing paths, matchings, cycles, and trees of maximum length, where the length od a cycle, matching, and tree, is the sum of the lengths of its edges.

Károlyi, Pach, and Tóth [6] show that if the edges of a complete geometric graph on $k^{2}+1$ points are colored red or blue, then there always exists a non-self intersecting red or blue path with $k+1$ edges.

The problem of finding non-crossing monotonic paths with many edges was first studied by Czyzowicz et al [5]. They proved that any labeled point set in convex position, contains a non-crossing monotonic path with at least $\sqrt{2 n}$ edges. This bound was improved recently by Sakai and Urrutia [7] to $\sqrt{3 n-3 / 4}-1 / 2$ by giving a simple proof for a result by Chung [2] concerning the length (number of elements) of a longest unimodal or anti-unimodal subsequences in a sequence.

For labeled point set $P$, we also study the problem of finding a non-crossing matching of $P$, not necessarily perfect, such that the sum of the weights of its edges is maximized, where the weight of an edge joining points $i$ and $j$ is the smallest of $\{i, j\}$, see Figure 1 .

We show that $P$ always has a matching of weight greater than $n^{2} / 6$ for $n \geq 2$. When the elements of $P$ are in convex position, then $P$ has a matching of weight at least $\left\lfloor n^{2} / 5\right\rfloor$. We observe that if we define the weight of the edge joining point $i$ to point $j$ to be the largest of $\{i, j\}$, and $P$ has $n=2 m$ elements, then we can always find a perfect matching with weight $(m+1)+\cdots+2 m$, which is the largest possible. To prove this, we recall a well known result that if a point set $S$ has $2 m$ elements (in general position), $m$ colored red, and $m$ colored blue, then 


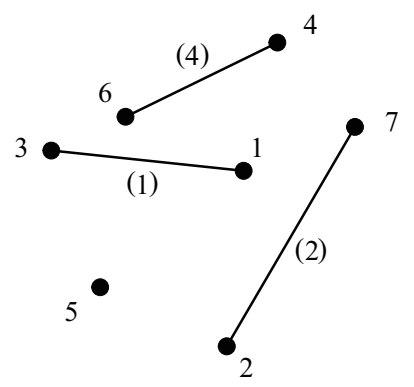

Fig. 1. A non-crossing matching with edges having weights 1, 2, 4 .

there always exists a non-crossing perfect matching of the elements of $S$, such that any edge in the matching has a blue and an red endpoint. Now color the elements of $P$ as follows: those elements whose label is smaller than or equal to $m$ are colored red, and the remaining points blue. Our result follows.

\section{The heaviest monotonic subsequences and paths}

In the rest of this paper, $P$ will denote a set of $n$ points in general position whose elements are labeled with the integers $\{1, \ldots, n\}$ in such a way that different points receive different labels. A point in $P$ labeled $i$ will be denoted as point $i$.

A path $\Pi$ of $P$ is a sequence of elements $i_{1}, \ldots, i_{k}$ of $P$ together with the closed line segments $\left[i_{r}, i_{r+1}\right]$ (called the edges of $P$ ) joining $i_{r}$ to $i_{r+1}, r=$ $1, \ldots, k-1$. We say that $\Pi$ is non-crossing if its edges do not cross each other. If in addition $i_{r}<i_{r+1}, 1 \leq r \leq k-1$, we call $\Pi$ a non-crossing increasing path of $P$. The weight of $\Pi$ is equal to $i_{1}+\cdots+i_{k}$.

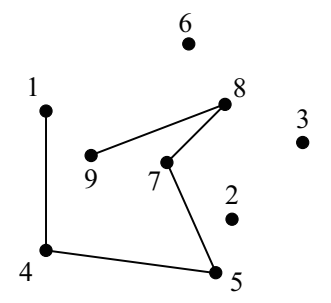

(a)

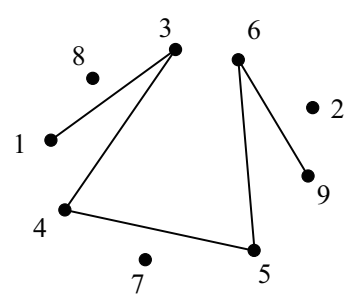

(b)

Fig. 2. (a) A non-crossing increasing path. (b) Finding the non-crossing increasing path with vertices $1,3,4,5,6,9$ is equivalent to finding the unimodal subsequence $3,6,9,5,4,1$ in the permutation $\{8,3,6,2,9,5,7,4,1\}$ (or the anti-unimodal subsequence $9,5,4,1,3,6$ in the permutation $\{9,5,7,4,1,8,3,6,2\})$. 
In this section we will study the following problem: Find the largest integer $\mathcal{W}(n)$ such that any labeled point set with $n$ elements has a non-crossing increasing path of weight at least $\mathcal{W}(n)$. In Figure $2(\mathrm{a})$, we show a non-crossing increasing path of weight 34 . The path with vertices $1,7,8,9$ is increasing, but has a crossing.

Observe that if the elements of $P$ are in convex position, that is, they are the vertices of a convex polygon, then the problem of finding a non-crossing increasing path of maximum weight, can be reduced to that of finding a unimodal or anti-unimodal subsequence of maximum weight in a permutation of $\{1, \ldots, n\}$ obtained from $P$, by reading its elements starting at some point of $P$, see Figure 2(b).

\subsection{The heaviest monotonic subsequence of a permutation}

Let $S=\{s(1), \ldots, s(n)\}$ be a permutation of $\{1, \ldots, n\}$. A subsequence of $S$ with elements $\left\{s\left(i_{1}\right), \ldots, s\left(i_{k}\right)\right\}$ is called an increasing subsequence if $s\left(i_{1}\right)<\cdots<$ $s\left(i_{k}\right)$, or a decreasing subsequence if $s\left(i_{1}\right)>\cdots>s\left(i_{k}\right)$. The subsequence is also called a monotonic subsequence if it is increasing or decreasing. The weight of $\left\{s\left(i_{1}\right), \ldots, s\left(i_{k}\right)\right\}$ is $s\left(i_{1}\right)+\cdots+s\left(i_{k}\right)$.

For each $s(i)$ of $S$, let us associate to it the point $\left(x_{i}, y_{i}\right)$ as follows: $x_{i}$ is the weight of the heaviest increasing subsequence of $S$ staring at $s(i)$, and $y_{i}$ is the weight of the heaviest decreasing subsequence of $S$ staring at $s(i)$. For example if $S=\{4,3,7,2,5,1,6\}$, then to $s(2)=3$, we associate the point $\left(x_{2}, y_{2}\right)=(3+5+6,3+2+1)=(14,6)$. It is easy to see that for indices $i<j$, if $s(i)<s(j)$, then $x_{i} \geq s(i)+x_{j}$, and if $s(i)>s(j)$, then $y_{i} \geq s(i)+y_{j}$. We now prove:

Theorem 1. Any permutation of $\{1, \ldots, n\}$ contains a monotonic subsequence of weight greater than $n \sqrt{n / 3}$. Our bound is asymptotically tight.

Proof. We now associate to each $s(i)$ the square $S Q(i)$ whose top-right vertex is the point $\left(x_{i}, y_{i}\right)$, and whose bottom-left vertex is the point $\left(x_{i}-s(i), y_{i}-s(i)\right)$.

We now prove that if $i \neq j$ then $S Q(i)$ and $S Q(j)$ have disjoint interiors. Suppose that $i<j$. Two cases arise. Suppose first that $s(i)<s(j)$. Then $x_{j} \leq$ $x_{i}-s(i)$, and $S Q(j)$ lies to the left of the vertical line with equation $x=x_{i}-s(i)$. If $s(i)>s(j)$ then $y_{j} \leq y_{i}-s(i)$, and $S Q(j)$ lies below the horizontal line $y=y_{i}-s(i)$. Thus in both cases $S Q(i)$ and $S Q(j)$ have disjoint interiors. See Figure 3 .

Let $R$ be a rectangle whose bottom-left vertex is the origin, and whose topright vertex is the point $(\alpha, \beta)$, where $\alpha$ is the maximum among all $x_{i}$ and $\beta$ is the maximum among all $y_{i}$. Since $R$ contains all the squares $S Q(i), i=1, \ldots, n$,

$$
\text { the area of } R=\alpha \beta \geq 1^{2}+2^{2}+\cdots+n^{2}>\frac{n^{3}}{3},
$$

and thus

$$
\max \{\alpha, \beta\}>n \sqrt{\frac{n}{3}}
$$




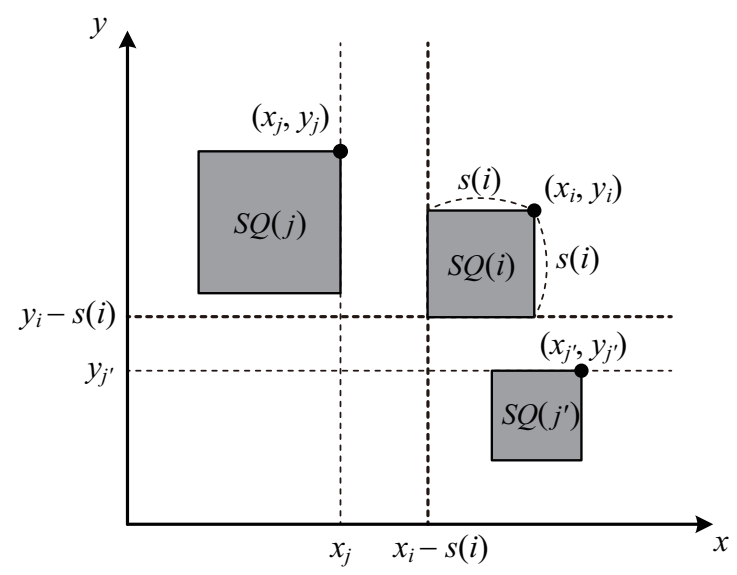

Fig. 3. $S Q(i)$ and $S Q(j), i \neq j$, have disjoint interiors.

Therefore for some $i, x_{i}$ or $y_{i}$ is greater than $n \sqrt{n / 3}$.

To see that our bound is asymptotically tight, we proceed as follows. Define $k$ and $m$ by $k=\sqrt[4]{4 n^{3} / 3}$, and $m=\sqrt{3 n} / 2$. Consider now the permutation $\Pi$ generated as follows:

$$
\begin{aligned}
& \lceil k\rceil,\lceil k\rceil-1, \ldots, 1,\lceil\sqrt{2} k\rceil,\lceil\sqrt{2} k\rceil-1, \ldots,\lceil k\rceil+1, \\
& \quad\lceil\sqrt{3} k\rceil,\lceil\sqrt{3} k\rceil-1, \ldots,\lceil\sqrt{2} k\rceil+1, \ldots, n, n-1, \ldots,\lceil\sqrt{m-1} k\rceil+1 .
\end{aligned}
$$

The permutation $\Pi$ consists of $m$ blocks of decreasing integers, such that for each of them, the sum of its elements is $n \sqrt{n / 3}+O(n)$. On the other hand, the heaviest increasing subsequence of $\Pi$, is the one containing the elements $\lceil k\rceil,\lceil\sqrt{2} k\rceil,\lceil\sqrt{3} k\rceil, \ldots, n$, which again has weight $n \sqrt{n / 3}+O(n)$. Our result follows.

We can now prove:

Theorem 2. Any labeled point set $P$ with $n$ elements has a non-crossing increasing path of weight greater than $n \sqrt{n / 3}$.

Proof. Assume that no two elements of $P$ lie on a vertical line, otherwise rotate $P$ slightly to achieve this condition. Project the elements of $P$ on the $x$-axis, and let $\Pi$ be the permutation of $\{1, \ldots, n\}$ obtained by reading the projections of the elements of $P$ on the $x$-axis from left to right. Then $\Pi$ has an increasing or deceasing subsequence of weight greater than $n \sqrt{n / 3}$. This subsequence induces a non-crossing increasing path of $P$ with weight greater than $n \sqrt{n / 3}$.

We conclude this section with general result concerning heaviest subsequences and paths. By arguing as in the proof of Theorems 1 and 2, we obtain: 
Theorem 3. (a) Any sequence $T=\left\{a_{1}, a_{2}, \ldots, a_{n}\right\}$ of $n$ distinct positive numbers contains a monotonic subsequence of weight at least $\sqrt{\sum_{i=1}^{n} a_{n}{ }^{2}}$.

(b) Any set of points in general position, labeled with elements of $T$, has a noncrossing increasing path of weight at least $\sqrt{\sum_{i=1}^{n} a_{n}^{2}}$.

\subsection{The heaviest increasing paths of point sets in convex position}

In this section we study the problem of finding a non-crossing increasing path of large weight for point sets in convex position. Our result will be based on the next result on unimodal or anti-unimodal subsequences of permutations.

Theorem 4. Any permutation $S$ of $\{1, \ldots, n\}$ contains a unimodal or antiunimodal subsequence of weight greater than $n \sqrt{2 n / 3}$.

Proof. The result follows immediately for $n \leq 3$. Thus assume $n \geq 4$. Let $S=$ $\{s(1), \ldots, s(n)\}$ be a permutation, and let $A=\sum_{i=1}^{n} s(i)^{2}$.

Lemma 1. There exist $j, 1 \leq j \leq n$, and $d$ with $|d| \leq n^{2} / 2$ such that

$$
s(1)^{2}+\cdots+s(j)^{2}=\frac{A}{2}-d \text { and } s(j+1)^{2}+\cdots+s(n)^{2}=\frac{A}{2}+d .
$$

Proof. Take the maximum $i$ such that $s(1)^{2}+\cdots s(i)^{2} \leq A / 2$. If in addition $\left(A-n^{2}\right) / 2 \leq s(1)^{2}+\cdots s(i)^{2}$, then we have only to let $j=i$. Thus assume that $s(1)^{2}+\cdots s(i)^{2}<\left(A-n^{2}\right) / 2$. In this case, we have $A / 2<s(1)^{2}+\cdots s(i)^{2}+$ $s(i+1)^{2}<\left(A+n^{2}\right) / 2$ by our choice of $i$ and by $0<s(i+1) \leq n$. Thus $j=i+1$ satisfies the desired property.

Let $j$ and $d$ be as in Lemma 1 , and define the sequences $S_{1}$ and $S_{2}$ by

$$
S_{1}=\{s(1), \cdots, s(j)\} \text { and } S_{2}=\{s(j+1), \cdots, s(n)\} .
$$

Denote by $\alpha_{1}$ (resp. $\beta_{1}$ ) the weight of the heaviest increasing (resp. decreasing) subsequence of $S_{1}$, and by $\alpha_{2}$ (resp. $\beta_{2}$ ) the weight of the heaviest increasing (resp. decreasing) subsequence of $S_{2}$. First note that $\alpha_{1}+\beta_{2}$ and $\beta_{1}+\alpha_{2}$ are weights of a unimodal subsequence and an anti-unimodal subsequence of $S$, respectively.

By arguing as in the proof of Theorem 1, we obtain

$$
\begin{aligned}
& \alpha_{1} \beta_{1} \geq s(1)^{2}+\cdots+s(j)^{2}=\frac{A}{2}-d, \text { and } \\
& \alpha_{2} \beta_{2} \geq s(j+1)^{2}+\cdots+s(n)^{2}=\frac{A}{2}+d .
\end{aligned}
$$


From these and the well known inequality $(x+y+z+w) / 4 \geq \sqrt[4]{x y z w}$ for positive numbers $x, y, z$ and $w$, it follows that

$$
\begin{aligned}
\left(\alpha_{1}+\beta_{2}\right)+\left(\beta_{1}+\alpha_{2}\right) & \geq 4 \sqrt[4]{\left(\alpha_{1} \beta_{1}\right)\left(\alpha_{2} \beta_{2}\right)} \\
& \geq 4 \sqrt[4]{(A / 2)^{2}-d^{2}} \\
& \geq 4 \sqrt[4]{\left(A^{2}-n^{4}\right) / 4}
\end{aligned}
$$

Since $A=n(n+1)(2 n+1) / 6>\left(n^{3}+n^{2}\right) / 3$, we have $A^{2}-n^{4}>n^{6} / 9$ for $n \geq 4$. Thus $\left(\alpha_{1}+\beta_{2}\right)+\left(\beta_{1}+\alpha_{2}\right)>2 n \sqrt{2 n / 3}$, and hence $\max \left\{\alpha_{1}+\beta_{2}, \beta_{1}+\alpha_{2}\right\}>$ $n \sqrt{2 n / 3}$, as desired.

At this point the best lower-bound we have for the weight of a unimodal or anti-unimodal subsequence of a permutation is approximately $2 n \sqrt{n / 3}$, and is given by the same permutation stated in the proof of Theorem 1 . On the other hand, the best upper-bound we have for the weight of a non-intersecting increasing path of the set of $n$ labeled points in convex position is approximately $n \sqrt{2 n}$. This is given by the following (circular) permutation for $n=2 k^{2}$ :

$$
\begin{array}{ccc}
n-k+1, & n-2 k+1, \ldots, k+1, & 1, \\
n-k+2, & n-2 k+2, \ldots, k+2, & 2, \\
\ldots, & \\
n=2 k^{2}, & n-k, \quad \ldots, 2 k, & k .
\end{array}
$$

It is easy to see that among all the unimodal or anti-unimodal subsequences of all possible permutations, a heaviest one is:

$$
n-k+1, n-k+2, \ldots, n, n-k, \ldots, 2 k, k,
$$

which has weight

$$
\frac{(2 n-k+1) k}{2}+\frac{n(2 k-1)}{2}<2 n k=n \sqrt{2 n} .
$$

Thus we have:

Theorem 5. Any set $P$ of $n$ labeled points in convex position has a non-crossing increasing path of weight greater than $n \sqrt{2 n / 3}$. Furthermore, there is a labeled point set in convex position such that any non-crossing increasing path has weight less than $n \sqrt{2 n}$.

Conjecture 1. Any set $P$ of $n$ labeled points in convex position has a non-crossing increasing path of weight greater than $n \sqrt{2 n}-O(n)$.

\section{The heaviest matchings}

In this section, we study the problem of finding non-crossing matchings of labeled point sets that maximize the sum of the weights of its edges. Recall that the weight of an edge joining point $i$ to point $j$, is the minimum of $\{i, j\}$. The sum of the weights of all edges of a matching is called the weight of the matching. 


\subsection{Point sets in convex position}

In this section, we consider the case where $P$ is in convex position. We start by observing that even if $P$ has $2 m$ elements, a non-crossing matching of maximum weight of $P$ is not necessarily a perfect matching. For example for the point set shown in Figure 4, the weight of any non-crossing perfect matching is 15.

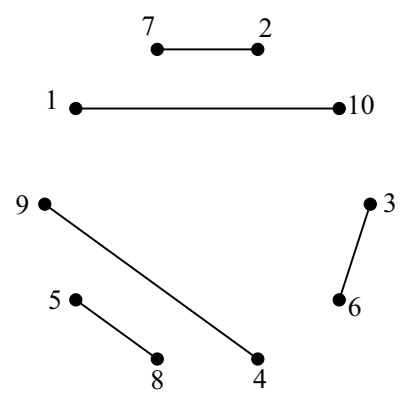

Fig. 4. The weight of the perfect matching shown is 15 . Moreover, any perfect matching of $P$ has weight 15 .

To prove this, observe that in any non-crossing perfect matching of the point set in Figure 4, any edge must leave an even number of points on each of its sides. Thus any such edge joins a point in $\{1,2,3,4,5\}$ to a point in $\{6,7,8,9,10\}$. It follows now that the weights of the edges of any non-crossing perfect matching $\mathcal{M}$ of $P$ are precisely $\{1,2,3,4,5\}$, and thus the weight of $\mathcal{M}$ is 15 . On the other hand, a non-perfect matching with non-crossing edges $\{[7,2],[9,10],[5,6],[8,4]\}$ has larger weight 20(>15).

This example can be generalized as follows: Let $P$ be an unlabeled point set with $2 \mathrm{~m}$ elements in convex position. We now color the elements of $P$ red or blue, in such a way that when we traverse the boundary of the convex hull of $P$ the colors of its elements alternate. Observe now that any edge of perfect matching $\mathcal{M}$ of $P$ joins a red to a blue point. Label the red and blue points of $P$ with the integers $\{1, \ldots, m\}$ and $\{m+1, \ldots, 2 m\}$, respectively. Then the weight of any edge of $\mathcal{M}$ belongs to $\{1, \ldots, m\}$. Since different edges in $\mathcal{M}$ have different weights, the weight of $\mathcal{M}$ is precisely $\left(\begin{array}{c}m+1 \\ 2\end{array}\right)$.

The maximum weight a non-crossing perfect matching of $P$ can have, is achieved when the elements of $P$ are labeled $1,2, \ldots, n$ in the clockwise order around the boundary of the convex hull of $P$. In this case, the weight of the heaviest matching of $P$ is precisely $1+3+\cdots+(2 m-1)=m^{2}$.

Since these bounds are valid for labeled point sets in general position, we have:

Lemma 2. The weight of any non-crossing perfect matching of a labeled point set $P$ in general position with $2 m$ elements is at least $\left(\begin{array}{c}m+1 \\ 2\end{array}\right)$ and at most $m^{2}$. These bounds are tight. 
Using similar arguments, we obtain:

Lemma 3. Let $Q$ be a set of $2 m$ points in general position whose elements are labeled with the integers $r+1, r+2, \ldots, r+2 m$. Then the weight of any perfect matching of $Q$ has the following bounds, and these bounds are tight:

- at least $(r+1)+(r+2)+\cdots+(r+m)=r m+\left(\begin{array}{c}m+1 \\ 2\end{array}\right)$, and

- at most $(r+1)+(r+3)+\cdots+[r+(2 m-1)]=r m+m^{2}$.

Next we consider matchings not necessarily perfect.

Lemma 4. Let $n_{1} \geq 0$ and $n_{2} \geq 1$ be integers, and $Q$ be a set of $2\left(n_{1}+n_{2}\right)$ points in convex position such that $2 n_{1}$ elements are colored red, and $2 n_{2}$ blue. Let $p$ be a blue element of $Q$. Then there is a non-crossing matching $\mathcal{M}$ of $Q$ such that the endpoints of each edge have the same color, and $\mathcal{M}$ matches all elements of $Q$, except at most two blue elements containing $p$.

Proof. We proceed by induction on $n=n_{1}+n_{2}$. The result follows immediately for $n=1$. Thus assume $n \geq 2$. We label the elements of $P$ as $p_{0}, \ldots, p_{2 n-1}$ around the boundary of the convex hull of $P$, where $p_{0}=p$. The indices are to be read modulo $2 n$. If $p_{i}$ and $p_{i+1}$ have the same color for some $i \not \equiv 0,-1$, then the edge $\left[p_{i}, p_{i+1}\right]$ together with edges of some matching $\mathcal{M}^{\prime}$ of $Q \backslash\left\{p_{i}, p_{i+1}\right\}$ form a matching $\mathcal{M}$ with the desired properties. Thus assume that the colors of $p_{1}, p_{2}, \ldots, p_{2 n-1}$ alternate (so $p_{1}$ and $p_{2 n-1}$ have a same color). Then $\mathcal{M}=$ $\left\{\left[p_{1}, p_{2 n-1}\right],\left[p_{2}, p_{2 n-2}\right], \cdots,\left[p_{n-1}, p_{n+1}\right]\right\}$ has the desired properties (and $p_{n}$ is colored blue).

We are now ready to prove the main result of this section.

Theorem 6. Let $P$ be a labeled point set with $n$ elements in convex position. Then the heaviest non-crossing matching of $P$ has weight at least $\left\lfloor n^{2} / 5\right\rfloor$.

Proof. To make our proof easy to understand, let us assume first that $P$ has $n=5 s$ elements.

To start, discard from $P$ all the elements with labels in $\{1, \ldots, s-2\}$. Now color with blue all the elements with labels in $\{s-1, \ldots, 3 s\}$, and with red all those with labels in $\{3 s+1, \ldots, 5 s\}$. By Lemma 4 , we can find matchings $\mathcal{M}^{\prime}$ and $\mathcal{M}^{\prime \prime}$ of $\{s-1, \ldots, 3 s\}$ and $\{3 s+1, \ldots, 5 s\}$, respectively, such that $\mathcal{M}=\mathcal{M}^{\prime} \cup \mathcal{M}^{\prime \prime}$ is non-crossing and $\mathcal{M}$ leave at most two elements of $\{s-1, \ldots, 3 s\}$, including $s-1$, unmatched.

First suppose that all the elements of $\{s-1, \ldots, 3 s\}$ are matched. Then by Lemma 3 with $r=s-2$ and $m=s+1$, the weight of $\mathcal{M}^{\prime}$ is at least

$$
(s-1)+s+\cdots+(2 s-2)+(2 s-1)=\frac{(3 s-2)(s+1)}{2} .
$$

Similarly, by applying Lemma 3 with $r=3 s$ and $m=s$, the weight of $\mathcal{M}^{\prime \prime}$ is at least:

$$
(3 s+1)+(3 s+2)+\cdots+(4 s-1)+4 s=\frac{(7 s+1) s}{2} .
$$


Adding up these summations, we get that the weight of $\mathcal{M}=\mathcal{M}^{\prime} \cup \mathcal{M}^{\prime \prime}$ is at least:

$$
5 s^{2}+s-1=\frac{n^{2}}{5}+\frac{n}{5}-1
$$

Next consider the case where two elements of $\{s-1, \ldots, 3 s\}$, including $s-1$, are unmatched. Let $t$ be the unmatched point other than $s-1$. Then the weight of $\mathcal{M} \cup\{[s-1, t]\}$ (which might have crossings) is at least $5 s^{2}+s-1$ in this case as well. Since the weight of $\mathcal{M}$ decreases by $s-1$ from the weight of $\mathcal{M} \cup\{[s-1, t]\}$, the weight of $\mathcal{M}$ is at least $5 s^{2}=n^{2} / 5$.

We can verify that this bound is valid for $n=5 s+k(1 \leq k \leq 4)$ as follows: We discard from $P$ the elements $1, \ldots, s+k-4$, and color the elements $s+k-3, \ldots, 3 s+k-2$ with blue, and the elements $3 s+k-1, \ldots, 5 s+k$ with red. Now we can argue as in the case where $n=5 s$ to see that $P$ has a non-crossing matching with weight at least $5 s^{2}+2 k s+k-1 \geq\left\lfloor(5 s+k)^{2} / 5\right\rfloor=\left\lfloor n^{2} / 5\right\rfloor$.

\subsection{Point sets in general position}

For point sets in general position, we have:

Theorem 7. Let $P$ be a labeled point set with $n \geq 2$ elements in general position. Then the heaviest non-crossing matching of $P$ has weight at least $L\left(n^{2}+\right.$ $n) / 6\rfloor$.

Proof. First consider the case where $n=3 s$. In this case, we discard from $P$ all the elements with labels $1, \ldots, s$. Then by Lemma 3 , any matching $\mathcal{M}$ of the remaining points has weight at least

$$
(s+1)+(s+2)+\cdots+2 s=\frac{(3 s+1) s}{2}=\frac{n(n+1)}{6} .
$$

We can argue similarly for the case where $n=3 s+1$ or $3 s+2$ by discarding from $P$ the elements with labels $1, \ldots, s+1$ or $1, \ldots, s$, respectively.

\section{References}

1. Alon N., Rajagopalan S., Suri S.: Long non-crossing congurations in the plane. Fundamenta Informaticae 22 (1995), 385-394.

2. Chung, F.R.K.: On unimodal subsequences. J. Combin. Theory Ser. A, 29 (1980) 267-279.

3. Dumitrescu A., Tóth C.: Long non-crossing configurations in the plane. Discrete Comput. Geom. 44 (2010) 727-752.

4. Erdős P., Szekeres G.: A combinatorial problem in geometry. Compositio Math. 2 (1935) 463-470.

5. Czyzowicz J., Kranakis E., Krizanc D., Urrutia J.: Maximal length common nonintersecting paths. Proc. Eighth Canadian Conference on Computational Geometry, August 1996, Ottawa, 180-189.

6. Károlyi G., Pach J., Tóth G.: Ramsey-type results for geometric graphs I. Discrete and Computational Geometry 18 (1997), 247-255. 
7. Sakai T., Urrutia J.: Monotonic polygons and paths of weighted point sets. Computational Geometry, Graphs and Applications. Lecture Notes in Computer Science Volume 7033, 2011, pp 164-175.

8. Tutte W.T.: The quest of the perfect square. Amer. Math. Monthly 72 (1965) 29-35. 